\title{
Prophylactic drainage of the abdominal cavity after surgery on the distal parts of the gastrointestinal tract
}

\section{Sopuev A. ${ }^{1}$, Abdiev A. ${ }^{2}$, Kalzhikeev A. ${ }^{3}$, Sydykov N. ${ }^{4}$, Mambetov A. ${ }^{5}$ \\ Профилактическое дренирование брюшной полости после операций \\ на дистальных отделах ЖКТ}

\author{
Сопуев А. А. ${ }^{1}$, Абдиев А. Ш. ${ }^{2}$, Калжикеев А. А. ${ }^{3}$, Сыдыков Н. Ж.. ${ }^{4}$ Мамбетов А. К. ${ }^{5}$ \\ ${ }^{1}$ Сопуев Андрей Асанкулович / Soриеv Andrei - доктор медицинких наук, профессор, \\ заведуюший кафедрой, \\ кафедра госпитальной хирургии, \\ Кыргызская государственная медииинская академия, г. Бишкек; \\ ${ }^{2}$ Абдиев Азамат Шакирович / Abdiev Azaтаt - кандидат медицинских наук, заведующий кафедрой, \\ кафедра хирургии, \\ Омский государственный медицинский институт, г. Ом; \\ ${ }^{3}$ Калжикеев Алмаз Абдрасулович / Kalzhikeev Almaz - врач-ординатор; \\ ${ }^{4}$ Сыдыков Нурлан Женишбекович / Sydykov Nurlan-кандидат медичинских наук, заведуюший, \\ приемное отделение, \\ Национальный хирургический центр; \\ ${ }_{5}^{5}$ Мамбетов Адыл Карагулович / Mambetov Adil - ассистент, \\ кафедра госпитальной хирургии, \\ Кыргызская государственная медицинская академия, г. Бишкек, Кыргызская Республика
}

\begin{abstract}
Аннотация: в статье на основе методов доказательной медицины определена эффективность профилактического дренирования брюшной полости после операций на дистальных отделах желудочнокишечного тракта путем сбора, интерпретации и интеграици надежных, важных и применяемых на практике доказательств, полученных в специальных медицинских исследованиях.

Abstract: on the basis of evidence-based medicine methods determined the effect of prophylactic drainage of the abdominal cavity after surgery on the distal parts of the gastrointestinal tract through the collection, interpretation and integration of reliable, critical and applied in practice, evidence obtained in special medical research.
\end{abstract}

Ключевые слова: профилактическое дренирование брюшной полости, рандомизированное клиническое исследование.

Keywords: prophylactic drainage of the abdominal cavity, a randomized clinical trial.

Профилактическое дренирование брюшной полости (ПДБП) после различных оперативных вмешательств широко практикуется в течение длительного времени в качестве эффективного метода профилактики осложнений в абдоминальной хирургии.

В течение последних 30-ти лет хирурги стали предпринимать усилия по оценке эффективности ПДБП после операций на органах брюшной полости в контролируемых рандомизированных клинических исследованиях (РКИ). Несмотря на полученные материалы, основанные на фактических данных о спорности ПДБП во многих случаях, большинство хирургов по всему миру продолжали использовать ПДБП на регулярной основе.

В связи с этим целью нашего исследования явилось на основе методов доказательной медицины определить эффективность ПДБП после операций на дистальных отделах желудочнокишечного тракта (ЖКТ) путем сбора, интерпретации и интеграции надежных, важных и применяемых на практике доказательств, полученных в специальных медицинских исследованиях.

Материал и методы исследования

На сегодняшний день опубликовано 8 РКИ, в которых была проведена оценка эффективности дренирования брюшной полости/полости малого таза после операций на колоректальном сегменте кишечника (Табл. 1). В большинстве исследований $[1,3,4,6,9,10,12]$ изучали только плановые хирургические вмешательства, только в 2-х РКИ в анализ были включены неотложные операции $[11,12]$. Количество экстренных хирургических вмешательств, вошедших в эти исследования, было небольшим. Эти больные в равной мере были представлены как в группе больных с дренированием брюшной полости, так и в группе больных без дренирования брюшной полости. Во всех исследованиях имела место схожая картина послеоперационных осложнений в обоих группах сравнения. В некоторых исследованиях отмечался более высокий уровень инфицированных послеоперационных ран у дренированных больных $[4,12,11]$, однако во второй по величине многоцентровой исследовательской серии, опубликованной в последнее время, отмечены сопоставимые уровни инфицирования послеоперационной раны в обоих группах сравнения $[9,10]$. Случаи несостоятельности анастомоза, распознанные клинически или рентгенологически в диапазоне от $1 \%$ до $23 \%$, имели место как у 
больных с дренированием брюшной полости, так и у больных без дренирования брюшной полости. Однако количественная разница между двумя наблюдаемыми группами не имела статистической достоверности. Различия в уровне смертности между наблюдаемыми группами в большинстве случаев не были связаны с несостоятельностью анастомозов.

Таблийа 1. Характеристика РКИ по оценке эффективности ПДБП

\begin{tabular}{|l|l|l|l|l|}
\hline Автор, год & $\begin{array}{l}\text { Уровень доказа- } \\
\text { тельности }\end{array}$ & $\begin{array}{l}\text { Коли- } \\
\text { чество } \\
\text { больных }\end{array}$ & Тип дренирования & $\begin{array}{l}\text { Продолжите } \\
\text { льность } \\
\text { дренирован } \\
\text { ия }\end{array}$ \\
\hline Hoffmann J. et al., 1987 [4] & $2 \mathrm{~b}$ & 60 & $\begin{array}{l}\text { Открытое } \\
\text { дренирование }\end{array}$ & 5 дней \\
\hline Johnson C.D. et al., 1989 [6] & $2 \mathrm{~b}$ & 106 & $\begin{array}{l}\text { Открытое } \\
\text { дренирование }\end{array}$ & 3 дня \\
\hline Hagmaeller E. et al., 1990 [3] & $2 \mathrm{~b}$ & 113 & Закрытое всасывание & $\begin{array}{l}\text { Не } \\
\text { сообщается }\end{array}$ \\
\hline Sagar P.M. et al., 1993 [11] & $1 \mathrm{~b}$ & 145 & Закрытое всасывание & 3 или 5 дней \\
\hline Sagar P.M. et al., 1995 [12] & $2 \mathrm{~b}$ & 100 & Закрытое всасывание & 7 дней \\
\hline Merad F. et al., 1998 [9] & $1 \mathrm{~b}$ & 317 & Закрытое всасывание & 5 дней \\
\hline Merad F. et al., 1999 [10] & $1 \mathrm{~b}$ & 494 & Закрытое всасывание & 5 дней \\
\hline Brown S.R. et al., 2001 [1] & $2 \mathrm{~b}$ & 59 & Закрытое всасывание & 3 дня \\
\hline
\end{tabular}

После объединения данных всех 8 РКИ материал для мета-анализа включил в себя 717 больных с профилактическим дренированием брюшной полости и 673 больных без профилактического дренирования брюшной полости. Сравнительный анализ показал небольшое преимущество в результатах по отношению к количеству возникших экссудативных внутрибрюшных жидкостных скоплений и раневой инфекции в группе больных без ПДБП, однако разница в полученных результатах не была статистически достоверной. Легочные осложнения были сопоставимы в обеих группах. Кроме того, мета-анализ, проведенный Urbach c coавт. [15], показал, что лишь в 1-м из 20-ти случаев несостоятельности анастомоза кишечное содержимое или гной стали выделяться через дренаж. Это указывает на то, что дренажи имеют низкую чувствительность (5\%) выявления несостоятельности анастомоза.

Учитывая разнородность сформированных анастомозов в 7-ми РКИ, задались задачей оценить эффективность ПДБП у больных после формирования тазовых и супрапромонториальных анастомозов. Анализ этих РКИ не выявил статистически достоверной разницы в количестве послеоперационных осложнений между группами пациентов с ПДБП и без ПДБП у больных с тазовыми и супрапромонториальными анастомозами.

\section{Обсуждение}

Только хорошо продуманные РКИ с адекватными размерами выборки могут предоставить убедительные данные по оценке эффективности ПДБП для снижения и выявления послеоперационных осложнений при хирургических вмешательствах в брюшной полости. Учитывая отсутствие окончательного обзора в этой области, мы провели многосторонний анализ на основе фактических данных для подготовки рекомендаций по использованию ПДБП в хирургии ЖКТ [5].

Результаты проведенного анализа показали, что использование рутинного ПДБП после неосложненных абдоминальных операций на колоректальном участке толстой кишки не дает каких-либо преимуществ [1, 3, 4, 6, 9-12]. Во многих случаях ПДБП бесполезно, увеличивает индекс болевых ощущений и общую стоимость лечебной процедуры. Имела место положительная тенденция в пользу бездренажной тактики по отношению к меньшему количеству таких осложнений, как послеоперационная раневая инфекция и клинически очевидная несостоятельность анастомоза. В исследованиях показано, что ПДБП обладает низкой чувствительностью при несостоятельности анастомозов и внутрибрюшных кровотечениях, ставя под сомнение предполагаемую функцию ПДБП $[2,5,7,8,13,14]$. Во всех РКИ имела место высокая вариабельность продолжительности ПДБП (2-7 суток). Это указывает на потребность в будущих РКИ, которые ориентированы на длительность ПДБП, особенно для краткосрочного ПДБП (24-48 часов), которое не изучалось. Исходя из этих исследований, мы приходим к выводу, что нет никаких доказательств того, что рутинное ПДБП в области колоректальных анастомозов оправдано в плановой хирургии. 
1. Brown S. R., Seon-Choen F. Eu. K. W., et al. A prospective randomised study of drains in infraperitoneal rectal anastomoses. Tech Coloproctol. 2001; 5: 89-92.

2. Greenall M. J., Evans M., Pollock A. V. Should you drain a perforated appendix? Br. J. Surg. 1978; 65: 880-882.

3. Hagmüller E., Lorenz D., Werthmann K., et al. Uses and risks of drainage following elective colon resection. A prospective, randomized and controlled clinical study. Chirurg. 1990; 61: 266-271.

4. Hoffmann J., Shokouh-Amiri M. H., Damm P., et al. A prospective, controlled study of prophylactic drainage after colonic anastomoses. Dis Colon Rectum. 1987; 30: 449-452.

5. Centre for Evidence-Based Medicine [Electronic resource]: http://www.cebm.net - (Accessed 2004).

6. Johnson C. D., Lamont P. M., Orr N., et al. Is a drain necessary after colonic anastomosis? J. R. Soc Med. 1989; 82: 661-664.

7. Liu C. L., Fan S. T., Lo C. M., et al. Abdominal drainage after hepatic resection is contraindicated in patients with chronic liver disease. Ann Surg. 2004; 239: 194-201.

8. Meakins J. L. Innovations in surgery: the rules of evidence. Am J Surg. 2002; 183: 399-405.

9. Merad F., Hay J.-M., Fingerhut A., et al. Is prophylactic pelvic drainage useful after elective rectal or anal anastomosis? A multicenter controlled randomized trial. Surgery. 1999; 125: 529-535.

10. Merad F., Yahchouchi E., Hay J.-M., et al. Prophylactic abdominal drainage after elective colonic resection and suprapromontory anastomosis. A multicenter study controlled by randomization. Arch Surg 1998; 133: 309-314.

11. Sagar P. M., Couse N., Kerin M., et al. Randomized trial of drainage of colorectal anastomosis. Br. J. Surg. 1993; 80: 769-771.

12. Sagar P. M., Hartley M. N., MacFie J., et al. Randomized trial of pelvic drainage after rectal resection. Dis Colon Rectum. 1995; 38: 254-258.

13. Sopuev A. A., Mamakeev K. M., Mamatov N. N., Abdiev A. Sh., Ovcharenko K. E., Akmatov T. A. An experimental evaluation of the effectiveness of dissociative anti-adhesive products. // International Journal Of Applied And Fundamental Research. - 2015. - № 1 - URL: www.science-sd.com/46024761 .

14. Sopuev A. A., Orozobekov B. K., Mamakeev J. B., Abdiev A. Sh., Akmatov T. A., Kalzhikeev A. A. Some aspects of diagnostics and treatment in chronic colonic stasis. // International Journal Of Applied And Fundamental Research. - 2015. - № 1 - URL: www.science-sd.com/460-24765.

15. Urbach D. R., Kennedy E. D., Cohen M. M. Colon and rectal anastomoses do not require routine drainage. Ann Surg. 1999; 229: 174-180. 